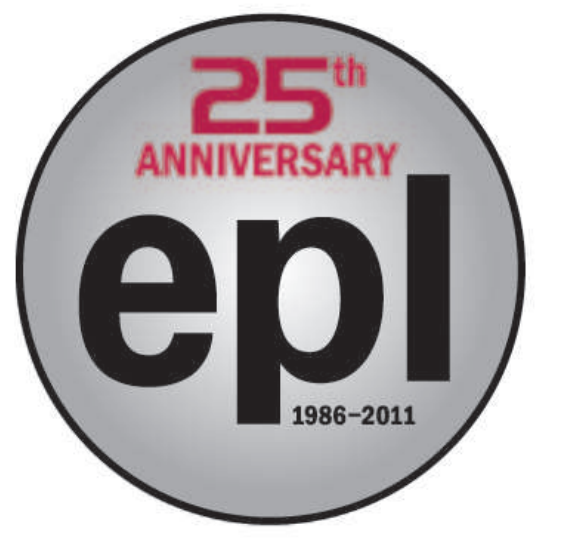

A LETTERS JOURNAL EXPLORING the FrontieRs of Physics

OFFPRINT

\title{
Tailoring the thermal Casimir force with graphene
}

V. Svetovoy, Z. Moktadir, M. Elwenspoek and H. Mizuta EPL, 96 (2011) 14006

Please visit the new website www.epljournal.org 


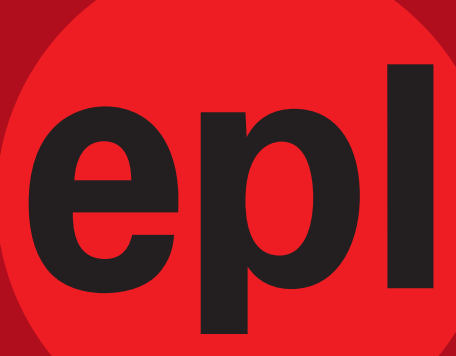

A LetTers JoUrnal EXPLoring the Frontiers of Physics

\section{AN INVITATION TO SUBMIT YOUR WORK}

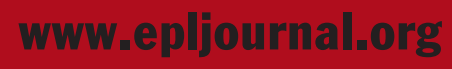

The Editorial Board invites you to submit your letters to EPL

EPL is a leading international journal publishing original, high-quality Letters in all areas of physics, ranging from condensed matter topics and interdisciplinary research

to astrophysics, geophysics, plasma and fusion sciences, including those with application potential.

The high profile of the journal combined with the excellent scientific quality of the articles continue to ensure EPL is an essential resource for its worldwide audience. EPL offers authors global visibility and a great opportunity to share their work with others across the whole of the physics community.

\section{Run by active scientists, for scientists}

EPL is reviewed by scientists for scientists, to serve and support the international scientific community. The Editorial Board is a team of active research scientists with an expert understanding of the needs of both authors and researchers.
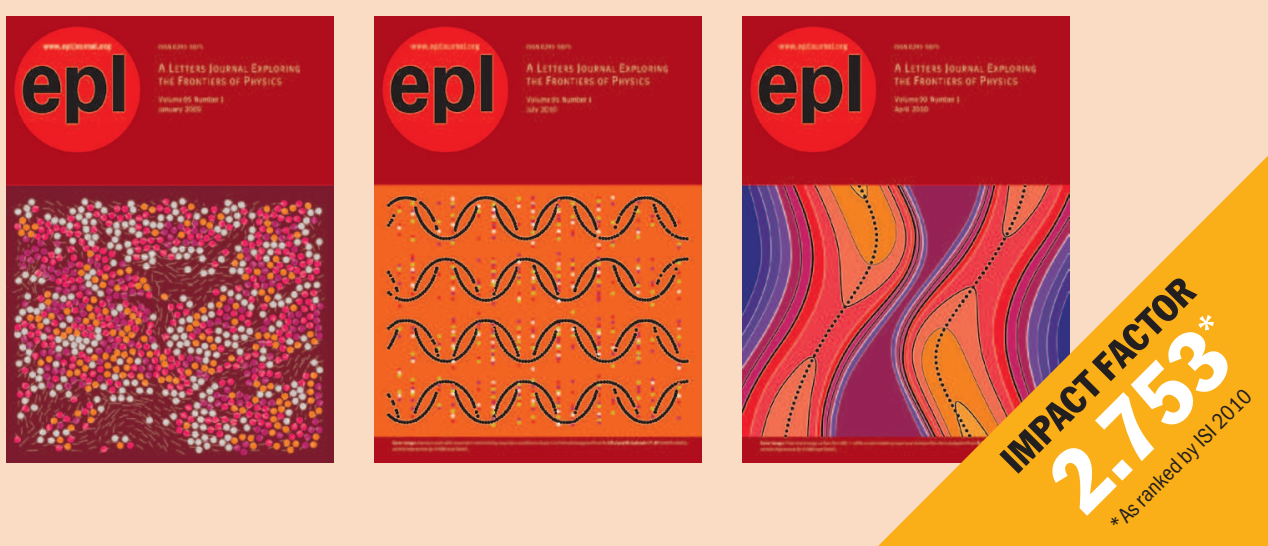

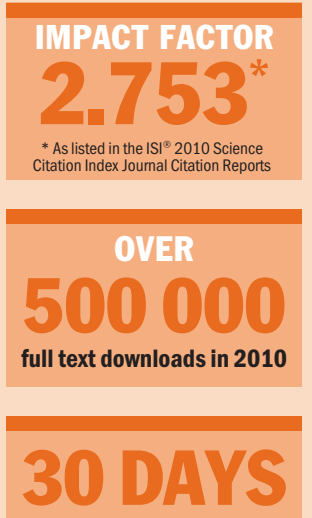

average receipt to online publication in 2010

\section{(1) $0 \longdiv { 6 }$ \\ citations in $\mathbf{2 0 1 0}$ \\ $37 \%$ increase from 2007}

"We've had a very positive experience with EPL, and not only on this occasion. The fact that one can identify an appropriate editor, and the editor is an active scientist in the field, makes a huge difference."

Dr. Ivar Martinv

Los Alamos National Laboratory, USA

\section{Six good reasons to publish with EPL}

We want to work with you to help gain recognition for your high-quality work through worldwide visibility and high citations.

Quality - The 40+ Co-Editors, who are experts in their fields, oversee the entire peer-review process, from selection of the referees to making all final acceptance decisions

Impact Factor - The 2010 Impact Factor is 2.753; your work will be in the right place to be cited by your peers

Speed of processing - We aim to provide you with a quick and efficient service; the median time from acceptance to online publication is 30 days

High visibility - All articles are free to read for 30 days from online publication date

International reach - Over 2,000 institutions have access to EPL, enabling your work to be read by your peers in 100 countries

Open Access - Articles are offered open access for a one-off author payment

Details on preparing, submitting and tracking the progress of your manuscript from submission to acceptance are available on the EPL submission website www.epletters.net.

If you would like further information about our author service or EPL in general, please visit www.epljournal.org or e-mail us at info@epljournal.org.

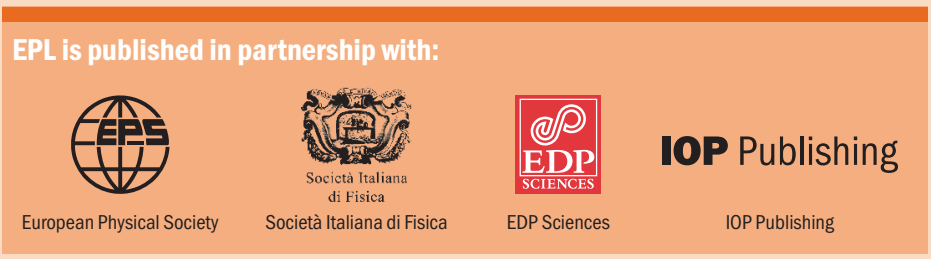

\section{www.epljournal.org}




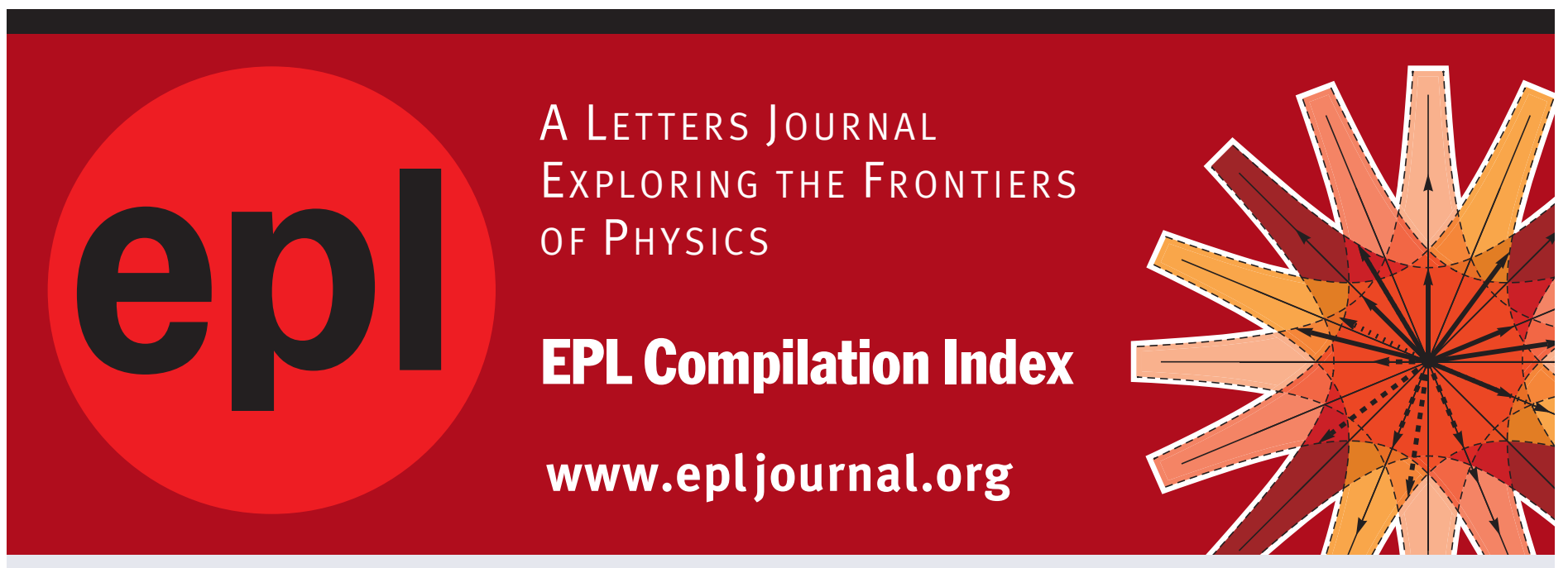

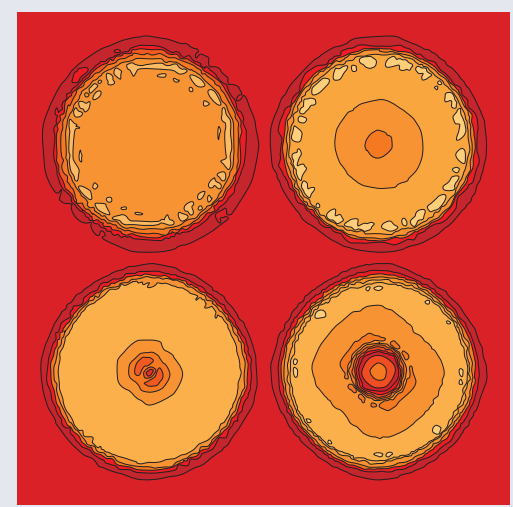

Biaxial strain on lens-shaped quantum rings of different inner radii, adapted from Zhang et al 2008 EPL 8367004.

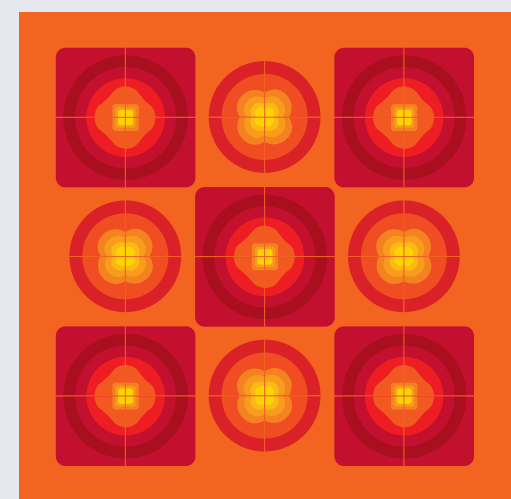

Artistic impression of electrostatic particle-particle interactions in dielectrophoresis, adapted from N Aubry and P Singh 2006 EPL 74623.

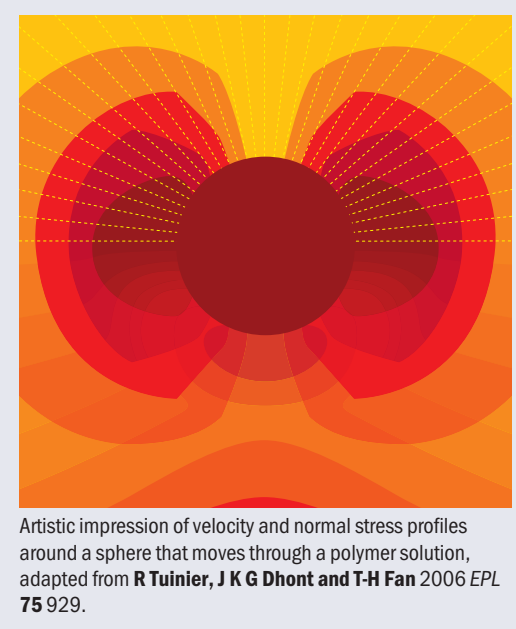

Visit the EPL website to read the latest articles published in cutting-edge fields of research from across the whole of physics.

Each compilation is led by its own Co-Editor, who is a leading scientist in that field, and who is responsible for overseeing the review process, selecting referees and making publication decisions for every manuscript.

- Graphene

- Liquid Crystals

- High Transition Temperature Superconductors

- Quantum Information Processing \& Communication

- Biological \& Soft Matter Physics

- Atomic, Molecular \& Optical Physics

- Bose-Einstein Condensates \& Ultracold Gases

- Metamaterials, Nanostructures \& Magnetic Materials

- Mathematical Methods

- Physics of Gases, Plasmas \& Electric Fields

- High Energy Nuclear Physics

If you are working on research in any of these areas, the Co-Editors would be delighted to receive your submission. Articles should be submitted via the automated manuscript system at www.epletters.net

If you would like further information about our author senvice or EPL in general, please visit www.epljournal.org or e-mail us at info@epljournal.org

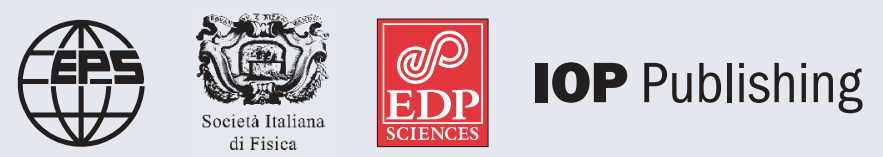

Image: Ornamental multiplication of space-time figures of temperature transformation rules (adapted from T. S. Bíró and P. Ván 2010 EPL 89 30001; artistic impression by Frédérique Swist). 


\title{
Tailoring the thermal Casimir force with graphene
}

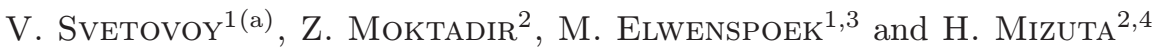 \\ ${ }^{1} \mathrm{MESA} \mathrm{A}^{+}$Institute for Nanotechnology, University of Twente - PO 217, 7500 AE Enschede, The Netherlands, EU \\ ${ }^{2}$ University of Southampton - Highfield, Southampton, SO17 1BJ, UK, EU \\ ${ }^{3}$ FRIAS, University of Freiburg - 79104 Freiburg, Germany, EU \\ ${ }^{4}$ School of Materials Science, Advanced Institute of Science and Technology (JAIST) - Ishikawa, 923-1292 Japan
}

received 25 June 2011; accepted in final form 19 August 2011

published online 20 September 2011

PACS 42.50.Lc - Quantum fluctuations, quantum noise, and quantum jumps

PACS 12.20.Ds - Quantum electrodynamics: Specific calculations

PACS 78.67.-n - Optical properties of low-dimensional, mesoscopic, and nanoscale materials and structures

\begin{abstract}
The Casimir interaction is omnipresent source of forces at small separations between bodies, which is difficult to change by varying external conditions. Here we show that graphene interacting with a metal can have the best known force contrast to the temperature and the Fermi level variations. In the distance range $50-300 \mathrm{~nm}$ the force is measurable and can vary a few times for graphene with a bandgap much larger than the temperature. In this distance range the main part of the force is due to the thermal fluctuations. We discuss also graphene on a dielectric membrane as a technologically robust configuration.
\end{abstract}

open áccess Copyright (c) EPLA, 2011

Introduction. - The Casimir force [1] manifests itself at short distances $(<1 \mu \mathrm{m})$ as a result of the electromagnetic interaction between neutral bodies without permanent polarizations. For two ideally reflecting parallel plates separated by a distance $a$, this force is given by: $F_{C}=$ $\left(\pi^{2} / 240\right)\left(\hbar c / a^{4}\right)$. The universal character of the force stimulated active development of the field [2] with applications in physics, biology, and technology.

The Lifshitz theory [3] gives the most detailed description of the force. According to this theory, current fluctuations (quantum and classical) in the bodies are responsible for the force. Fluctuations in a wide range of frequencies give significant contribution to the force. For this reason it is difficult to change the force at will as one has to modify the dielectric response of interacting materials in a wide range of frequencies. Hydrogen-switchable mirrors did not show observable contrast to the Casimir force [4]. It was demonstrated that the force between indium tin oxide (ITO) and a gold surface is $50 \%$ smaller than it is between two Au surfaces [5]. For the same material the best result was found for the phase-changing material (Ag-In-Sb-Te) with $20 \%$ difference between amorphous and crystalline phases [6]. In situ modulation of the force between a gold sphere and a silicon membrane [7] was shown to $1 \%$ level when the carrier density was changed optically by 4 orders of magnitude.

\footnotetext{
(a) E-mail: v.svetovoy@utwente.nl
}

The force measured in modern experiments is mainly the result of quantum fluctuations whilst the force due to classical fluctuations (thermal Casimir or Lifshitz force) was measured only recently between an ultracold atomic cloud and a sapphire substrate [8], and between two Au surfaces [9]. The thermal fluctuations dominate the force at large distances $a \gtrsim \hbar c / T\left(k_{B}=1\right)$ where the force itself is extremely weak and approaches the Lifshitz limit [3]. Between two metals this limit is given by

$$
F_{L}=\frac{T \zeta(3)}{8 \pi a^{3}}, \quad a \gg \lambda_{T}=\frac{\hbar c}{T}
$$

where $\lambda_{T}$ is the thermal wavelength and $\zeta(x)$ is the zetafunction.

In this paper we show that significant variation (up to 5 times) of the total Casimir force is possible for graphene with a bandgap $2 \Delta \gg T$. The force changes in response to the variation of the Fermi level mainly due to the change of its thermal part. It can be realized at the distance range $a=50-300 \mathrm{~nm}$, where the force is well measurable.

Graphene, a single layer material with carbon atoms arranged in a honeycomb lattice, attracted enormous attention [10,11]. Unusual electronic properties of graphene are due to massless relativistic dispersion of electrons at low energies [11,12]. The Casimir/van der Waals interaction of graphene was mainly discussed at zero temperature [13-15] with the conclusion that the force due 
to graphene is weak in comparison with the interaction of bulk bodies.

An important development was made by GómezSantos [16] at finite temperature. It was argued that at $T=0$ graphene is a critical system, with no characteristic length scale. At non-zero $T$ this scale is given by the thermal length $\xi_{T}=\hbar v_{F} / T$, where $v_{F} \approx 10^{6} \mathrm{~m} / \mathrm{s}$ is the Fermi velocity in graphene. It was found that in the long distance limit the force between two graphene sheets is given by the same eq. (1) but this equation is true for much shorter distances $a \gg \xi_{T}$. At room temperature the scales $\xi_{T}$ and $\lambda_{T}$ are $25 \mathrm{~nm}$ and $7.6 \mu \mathrm{m}$, respectively. This property makes the thermal Casimir force operative for separations in the $50-300 \mathrm{~nm}$ range, which are readily accessible using an atomic force microscope (AFM) or other force measuring techniques (see [2] for a review).

Graphene is a promising material for the development of high-performance electronic devices [17] but pristine graphene is a semimetal with zero bandgap [11]. The major challenge of graphene electronics is to open an energy bandgap [18]. As we will see later, the bandgap is also important for tailoring the Casimir force by electronic means. Significant progress has been made in this direction. For instance, epitaxially grown graphene on $\mathrm{SiC}$ has a gap of $2 \Delta \approx 0.26 \mathrm{eV}$ [19]. Opening a bandgap was also demonstrated by water adsorption [20] and patterned hydrogen adsorption [21]. Graphene nanomesh proved to generate bandgaps with values depending on the mesh density [22-24]. Very recently, an efficient way to fabricate graphen nanomesh was developed [25]. In the present work we will assume the presence of a gap without specifying its origin.

Graphene on a substrate. - We consider here the interaction between two plates 1 and 2 having dielectric functions $\varepsilon_{1}(\omega)$ and $\varepsilon_{2}(\omega)$, respectively. In contrast with [16] graphene is not free standing but covers the plate 1 . As we will see it has significant influence on the system. The case of suspended graphene is reproduced by taking $\varepsilon_{1}(\omega)=1$. The Lifshitz formula [3] expresses the force between two parallel plates via their reflection coefficients. If graphene sheet has the two-dimensional (2D) dynamical conductivity $\sigma$, then the reflection coefficient of the plate with graphene (for $p$ polarization) is given by $[26,27]$

$$
r_{1}=\frac{k_{0} \varepsilon_{1}-k_{1}+(4 \pi \sigma / \omega) k_{0} k_{1}}{k_{0} \varepsilon_{1}+k_{1}+(4 \pi \sigma / \omega) k_{0} k_{1}} .
$$

Here the normal components of the wave vectors in vacuum and in the substrate are $k_{0}=\sqrt{\omega^{2} / c^{2}-q^{2}}$ and $k_{1}=\sqrt{\varepsilon_{1} \omega^{2} / c^{2}-q^{2}}$, respectively, where $\mathbf{q}$ is the wave vector along the plate. In the $T=0$ limit the graphene conductivity is $\sigma \sim e^{2} / \hbar$ for frequencies up to near UV $[26,28,29]$. It means that the reflection coefficient gets only a small correction $\sim \alpha=e^{2} / \hbar c=1 / 137$ due to the presence of graphene on the dielectric substrate. This explains a weak force between two graphene sheets $[13,14]$ (2.6\% of the force between ideal metals, $\sim \pi \alpha$ ).
In this paper we neglect the effects due to $\alpha$ on the force. In this approximation the force between a suspended graphene sheet and any another material tends to zero at $T=0$ (negligible in comparison with the force between bulk materials). If graphene covers a substrate then the force difference $\Delta F=F_{g}-F_{b}$ is equally negligible, where $F_{g}$ and $F_{b}$ are the force with and without the graphene layer on the substrate, respectively. One can systematically neglect the effects $\sim \alpha$ in $\Delta F$ by taking the non-retarded limit $c \rightarrow \infty$. The possibility to use this limit was already indicated for two graphene sheets [16]. Detailed calculation of the force between suspended graphene and $\mathrm{Au}$ [30] gave an independent proof of this approximation. Taking the limit $c \rightarrow \infty$ in the Lifshitz formula one finds the graphene contribution:

$$
\Delta F(a, T)=\frac{T}{8 \pi a^{3}} \sum_{n=0}^{\infty} \int_{\xi_{n}}^{\infty} \mathrm{d} x x^{2}\left[\frac{R}{e^{x}-R}-\frac{R_{0}}{e^{x}-R_{0}}\right],
$$

where the integration variable in the physical terms is $x=2 a q$. Here $R=r_{1} r_{2}$ is the product of the reflection coefficients for the body 1 (covered with graphene) and the body 2 , and $R_{0}=r_{0} r_{2}$, where $r_{0}$ is the reflection coefficient of the body 1 without graphene. The reflection coefficients also have to be calculated in the non-retarded limit. The sum is taken over the imaginary Matsubara frequencies $\omega_{n}=2 i \pi T n / \hbar$, which enter the dielectric functions in the reflection coefficients. Only $p$ polarization contributes to $\Delta F$ since the $s$ polarization vanishes in the non-retarded limit. It has to be stressed that $c \rightarrow \infty$ limit can only be applied to the force difference but not to $F_{g}$ or $F_{b}$ separately. We keep the lower integration limit in (3) finite $\xi_{n}=2 \pi T n(\hbar c / 2 a)^{-1}$. Doing so we stay within acceptable uncertainty $\sim \alpha$ in $\Delta F$. This definition is more convenient because convergence of $\Delta F$ is defined only by graphene but not high frequency transparency of the bulk bodies.

To proceed further we need to know the dielectric function of graphene. It is related to the dynamical conductivity of the vacuum-graphene-dielectric system by the relation [31]

$$
\varepsilon(q, \omega)=1+\frac{4 \pi \sigma(q, \omega)}{\omega}\left(\frac{k_{0} k_{1}}{\varepsilon_{1} k_{0}+k_{1}}\right) .
$$

Combining eq. (4) with eq. (2) one finds a simple expression for the reflection coefficient of the body covered with graphene:

$$
r_{1}=1-\frac{1-r_{0}}{\varepsilon(q, \omega)}
$$

Dielectric function of graphene. - The dielectric function of graphene can be calculated using the random phase approximation (RPA). The RPA was used extensively for graphene in different situations (see the reviews $[11,12])$. Specific to our case, we need to know this function for imaginary frequencies at non-zero temperature for doped graphene with a non-zero gap. In the literature one can find $\varepsilon(q, \omega)$ only in different limiting cases. 


$$
I_{1,2}=\int_{0}^{\infty} \mathrm{d} \mu \int_{0}^{\pi} \mathrm{d} \nu\left[1 \mp \frac{Q^{2}\left(\xi^{2}+\eta^{2}-2\right)+\Delta_{T}^{2}}{\epsilon_{1} \epsilon_{2}}\right] \frac{Q\left(\epsilon_{2} \pm \epsilon_{1}\right)\left(\xi^{2}-\eta^{2}\right)}{4 Z^{2}+\left(\epsilon_{2} \pm \epsilon_{1}\right)^{2}}\left[\frac{\sinh \epsilon_{2}}{\cosh \epsilon_{2}+\cosh \epsilon_{F}} \pm \frac{\sinh \epsilon_{1}}{\cosh \epsilon_{1}+\cosh \epsilon_{F}}\right]
$$

For non-zero gap the electron energy in the valence $(s=-1)$ or in the conduction $(s=+1)$ band is $E_{s \mathbf{k}}=$ $s \sqrt{\left(\hbar v_{F} \mathbf{k}\right)^{2}+\Delta^{2}}$. The probability to find an electron (hole) with the energy $E_{s \mathbf{k}}$ is given by the Fermi distribution $f_{s \mathbf{k}}=\left[1+e^{\left(E_{s k}-E_{F}\right) / T}\right]^{-1}$, where $E_{F}$ is the Fermi level. In the RPA, the dielectric function of graphene can be expressed as $\varepsilon=1+v_{c}(q) \Pi(q, \omega)$. Here $v_{c}=2 \pi e^{2} / \kappa q$ is the $2 \mathrm{D}$ Coulomb interaction, $\kappa$ is defined by the environment of the graphene layer (in our case $2 \kappa=\varepsilon_{1}(0)+1$ ), and $\Pi(q, \omega)$ is the $2 \mathrm{D}$ polarizability given by the bare bubble diagram:

$$
\Pi(q, \omega)=-4 \sum_{s, s^{\prime}} \int \frac{\mathrm{d}^{2} k}{(2 \pi)^{2}} V_{k k^{\prime}}^{s s^{\prime}} \frac{f_{s \mathbf{k}}-f_{s^{\prime} \mathbf{k}^{\prime}}}{\hbar \omega+E_{s \mathbf{k}}-E_{s^{\prime} \mathbf{k}^{\prime}}},
$$

where $\mathbf{k}^{\prime}=\mathbf{k}+\mathbf{q}, s, s^{\prime}= \pm 1$, and the vertex factor is given by $2 V_{k k^{\prime}}^{s s^{\prime}}=1+\left(\hbar^{2} v_{F}^{2} \mathbf{k} \cdot \mathbf{k}^{\prime}+\Delta^{2}\right) / E_{s k} E_{s^{\prime} k^{\prime}}$. The factor 4 at the front comes from two spins and two valleys degeneracy.

In what follows we use the dimensionless variables:

$$
Q=\frac{\hbar v_{F} q}{2 T}, \quad Z=\frac{\hbar \zeta}{2 T}, \quad \Delta_{T}=\frac{\Delta}{T}, \quad \epsilon_{F}=\frac{E_{F}}{T},
$$

where $\zeta$ is the imaginary frequency. It is convenient to calculate the polarizability in the elliptic coordinates $\mu$ and $\nu$ defined by the relations:

$$
k=\frac{q}{2}(\cosh \mu-\cos \nu), \quad k^{\prime}=\frac{q}{2}(\cosh \mu+\cos \nu) .
$$

The notations $\xi=\cosh \mu$ and $\eta=\cos \nu$ will also be used. Separating interband ( $\mathbf{k}$ and $\mathbf{k}^{\prime}$ in different bands) and intraband (k and $\mathbf{k}^{\prime}$ in one band) transitions in (6) we can present the dielectric function as

$$
\varepsilon(q, i \zeta)=1+\frac{\alpha_{g}}{\pi}\left(I_{1}+I_{2}\right), \quad \alpha_{g}=\frac{e^{2}}{\kappa \hbar v_{F}},
$$

where $I_{1}$ and $I_{2}$ are the contributions coming from interband and intraband transitions, respectively, and $\alpha_{g}$ is the interaction constant in graphene. For $I_{1,2}$ one finds

$$
\text { see eq. (10) above }
$$

In eq. (10) $\epsilon_{1,2}=\sqrt{Q^{2}(\xi \mp \eta)^{2}+\Delta_{T}^{2}}$ and the upper (lower) sign is related to index $1(2)$.

Typical values of $q$ for the Casimir problem are $\sim 1 / 2 a$. Therefore, for the distances $a \gg \xi_{T}$ of interest in this paper, the values of $Q$ are always small, i.e. $Q \ll 1$. In this limit eq. (10) can be simplified further. The parameter $Q \eta$ is always small but $Q \xi$ is not. In fact, the important values of $\xi$ in the integrals are large: $\xi \sim \max \left(1 / Q, \Delta_{T} / Q\right)$. Making the corresponding expansions and performing explicit integrations over $\nu$ we find for $I_{1,2}$ in the limit $Q \ll 1:$

$$
\begin{aligned}
I_{1}= & \pi Q \int_{\Delta_{T}}^{\infty} \mathrm{d} \epsilon \frac{\epsilon^{2}+\Delta_{T}^{2}}{\epsilon^{2}\left(Z^{2}+\epsilon^{2}\right)} \cdot \frac{\sinh \epsilon}{\cosh \epsilon+\cosh \epsilon_{F}}, \\
I_{2}= & \frac{2 \pi}{Q} \int_{\Delta_{T}}^{\infty} \mathrm{d} \epsilon \epsilon\left[1-\frac{Z}{\sqrt{Z^{2}+Q^{2}-\left(\Delta_{T} Q / \epsilon\right)^{2}}}\right] \\
& \times \frac{1+\cosh \epsilon \cosh \epsilon_{F}}{\left(\cosh \epsilon+\cosh \epsilon_{F}\right)^{2}},
\end{aligned}
$$

where we introduced a new integration variable $\epsilon=$ $\sqrt{Q^{2} \xi^{2}+\Delta_{T}^{2}}$. Note that the intraband contribution dominates the dielectric function in the $Q \ll 1$ limit.

The force. - In the large distance limit $a \gg \xi_{T}$ the dielectric function of graphene is significant $(\varepsilon-1 \gg \alpha)$ at frequencies $\hbar \zeta \lesssim T$, which are low for $T \sim 300 \mathrm{~K}$. For these frequencies most of dielectric materials have static permittivities and metals can be considered as perfect conductors. In such cases we can simplify the calculation of $\Delta F$ in eq. (3) taking the static permittivities $\varepsilon_{1,2}(0)$ for bulk bodies $\left(\varepsilon_{2}(0) \rightarrow \infty\right.$ for metals) and keeping $q$ and $\zeta$ dependence only for the graphene dielectric function $\varepsilon(q, i \zeta)$. It has to be mentioned that $\varepsilon(q, i \zeta)$ is essentially nonlocal. This nonlocality, however, is two-dimensional, which simplifies the calculation of the Casimir force in comparison with the $3 \mathrm{D}$ case [32]. This is because there is only an in-plane wave vector.

Consider first a gapless graphene. For $\Delta=0$ the dielectric function at large distances $a \gg \xi_{T}$ follows from (9) and (12)

$$
\varepsilon(q, i \zeta)=1+\frac{2 \alpha_{g} G\left(\epsilon_{F}, 0\right)}{Q}\left(1-\frac{Z}{\sqrt{Z^{2}+Q^{2}}}\right) .
$$

The function $G\left(\epsilon_{F}, 0\right)$ here increases monotonously starting from $2 \ln 2$ at $\epsilon_{F}=0$ (fig. 1(a)). In general $G(x, y)$ is given by the expression

$$
G(x, y)=\int_{y}^{\infty} \mathrm{d} t t \frac{1+\cosh t \cosh x}{(\cosh t+\cosh x)^{2}} .
$$

Let us stress that the characteristic frequency in the dielectric function (13) is $\zeta \sim v_{F} q$ as is expected from general consideration [16]. For the Matsubara frequency $\omega_{0}=i 0$ the dielectric function has a metallic character, i.e. $\varepsilon(q, i 0) \gg 1$ and the reflection coefficient of the body covered with graphene approaches 1 , i.e. $r_{1} \rightarrow 1$. Already, for $n=1$ we have $Z_{1} \gg Q$ and $\varepsilon\left(q, i \zeta_{1}\right)$ is strongly suppressed. For $n \neq 0$ the reflection coefficient approaches 

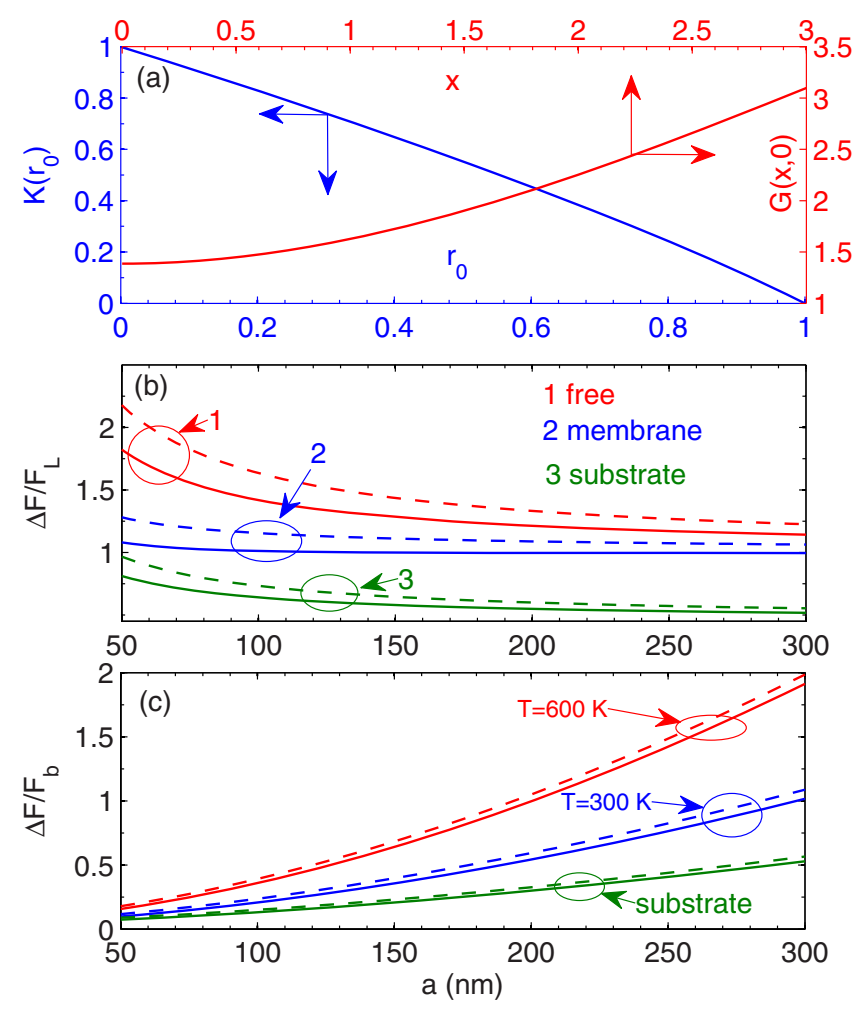

Fig. 1: (Color online) (a) (top-right axes) Function $G(x, 0)$ that enters eq. (13). (bottom-left axes) The factor $K\left(r_{0}\right)$ in eq. (15) as a function of the static reflection coefficient of the substrate supporting graphene. (b) The force ratio $\Delta F / F_{L}$ as a function of distance for free-standing graphene, grapheneon-membrane, and graphene-on-substrate at $T=300 \mathrm{~K}$. The solid lines are for $\epsilon_{F}=0$ and the dashed lines are for $\epsilon_{F}=10$. (c) The relative force for graphene-on-membrane as a function of $a$ for two different temperatures. The lowest dashed and solide curves are for graphene-on-substrate at $T=300 \mathrm{~K}$. The curves for membrane in (b) and (c) were calculated for $h=$ $20 \mathrm{~nm}$ and $r_{0}=0.6$.

the substrate value, $r_{1} \rightarrow r_{0}$. Let us stress that just one monolayer covering the substrate makes it perfectly reflecting at low frequencies.

For distances $a \gg \xi_{T}$ we can apply (13) to calculate the force (3). The $n=0$ term dominates in $\Delta F$. If the second body is a metal we can take $R=1$ and $R_{0}=r_{0}$, where $r_{0}$ has to be taken in the static limit. The force in this case is

$$
\Delta F(a, T)=\frac{T \zeta(3)}{8 \pi a^{3}} K\left(r_{0}\right), \quad a \gg \frac{\hbar v_{F}}{T},
$$

where the function $K\left(r_{0}\right)$ describes the effect of the substrate on the force. This function is shown in fig. 1(a) and is expressed analytically as

$$
K\left(r_{0}\right)=\frac{1}{2 \zeta(3)} \int_{0}^{\infty} \mathrm{d} x x^{2}\left[\frac{1}{e^{x}-1}-\frac{r_{0}}{e^{x}-r_{0}}\right] .
$$

For suspended graphene $r_{0}=0$, and eq. (15) coincides with the Lifshitz force $F_{L}$. Note that a metallic substrate for graphene will result in the zero force because $K\left(r_{0}\right) \rightarrow 0$ when $r_{0} \rightarrow 1$.

The effect of graphene will be appreciable if $\Delta F$ is measurable but also if $\Delta F$ is not negligible in comparison with the background force $F_{b}$. The force (15) is maximal for free-standing graphene when $F_{b}=0$. This configuration is realizable in practice [33] and has significant interest. However, it can not always be practical due to the deformation induced by the force. A more stable configuration is graphene on a dielectric membrane of thickness $h$. For a membrane, the reflection coefficient is

$$
r_{0 m}=r_{0} \frac{1-e^{-2 q h}}{1-r_{0}^{2} e^{-2 q h}}
$$

where $r_{0}$ corresponds to the bulk material. For a thin membrane, $h \ll a, r_{0 m}$ becomes small and the background force $F_{b}$ is much weaker than that for the thick substrate. For graphene-on-membrane the force in the long distance limit is also given by eq. (15) but now the factor $K$ depends slightly on the distance due to $q$-dependence of $r_{0 m}$. The graphene-on-membrane configuration maximizes not only the absolute value of the force $\Delta F$ but also the relative value $\Delta F / F_{b}$. This is an important practical observation.

Figure 1(b) shows how the force approaches its limit value (15) for free-standing graphene, for graphene on $20 \mathrm{~nm}$ thick $\mathrm{SiO}_{2}$ membrane, and for graphene on a thick $\mathrm{SiO}_{2}$ substrate. Numerical calculations were performed using the dielectric function (9) with $I_{1,2}$ from (10) without additional approximations. The continuous lines are for $E_{F}=0$ and the dashed lines are for $E_{F}=10 T$. One can see that the force is not very sensitive to $E_{F}$.

This is especially obvious in fig. 1(c) where the relative force $\left(\Delta F\right.$ in respect to the background force $\left.F_{b}\right)$ is shown. This figure demonstrates significant dependence on temperature and shows that the relative force is considerably smaller for a thick substrate than for a thin membrane.

Significant dependence on the Fermi level is desirable to change the force by electronic means. This can be realized if graphene has a non-zero gap. The material will change from insulating to conducting state in response to the position of $E_{F}$. It has to influence the dielectric function and thus the force. The dielectric function of graphene with the gap $2 \Delta$ was calculated in [34], on the real frequency axis at $T=0$. Here we are using our result $(9),(10)$ for the dielectric function on the imaginary frequency axis at non-zero $T$.

As in the case of gapless graphene the main contribution to the force at large distances comes from the $n=0$ term, which depends on the static dielectric function:

$$
\varepsilon(q, i 0)=1+\frac{2 \alpha_{g}}{Q} G\left(\epsilon_{F}, \Delta_{T}\right),
$$

where the function $G\left(\epsilon_{F}, \Delta_{T}\right)$ is given by eq. (14). The gap gives significant effect for $\Delta_{T} \gg 1$. If the Fermi level is in the middle of the gap, i.e. $\epsilon_{F}=0$, the function $G$ 

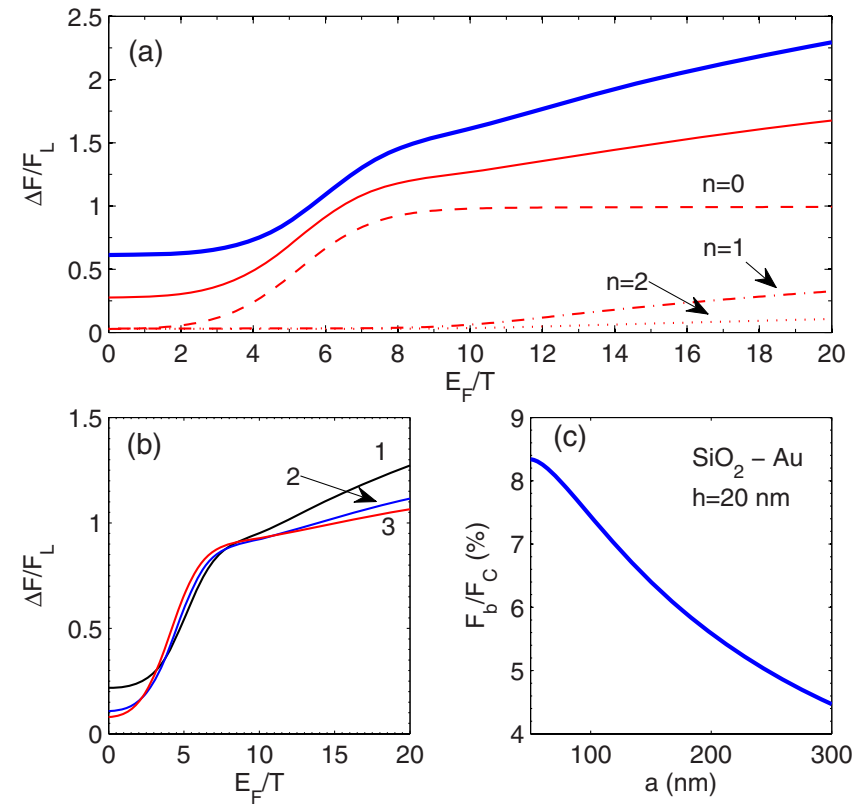

Fig. 2: (Color online) (a) The force as a function of the Fermi level for free-standing graphene with $\Delta_{T}=10$. The thick line is for $a=50 \mathrm{~nm}$ and the thin solid line is for $a=$ $100 \mathrm{~nm}$. The dashed, dash-dotted, and dotted lines are the first three components for the $a=100 \mathrm{~nm}$ case. (b) The force for graphene-on-membrane $\left(\Delta_{T}=10\right)$. The lines marked as 1,2 , and 3 correspond to $a=100,200$, and $300 \mathrm{~nm}$, respectively. (c) The background force as a function of the distance in units of the bare Casimir force $F_{C}=\pi^{2} \hbar c / 240 a^{4}$.

is exponentially suppressed, i.e. $G\left(0, \Delta_{T}\right) \approx 2 \Delta_{T} e^{-\Delta_{T}}$. In this case the effect of graphene on the force is small. When $\epsilon_{F}$ becomes comparable with $\Delta_{T}$ the dielectric function $\varepsilon(q, i 0)$ is large and the effect of graphene is significant. In the long distance limit the force behavior is similar to eq. (15).

Figure 2(a) shows the force for suspended graphene with the gap $\Delta_{T}=10$ as a function of the Fermi level for $a=50 \mathrm{~nm}$ and $100 \mathrm{~nm}$ (solid curves). About ten terms are important in the sum (3); the first three terms for $a=100 \mathrm{~nm}$ are shown. Indeed, the $n=0$ term gives the main contribution. The finite value of the force at $E_{F}=0$ decreases as $a$ and $\Delta$ increase. It is mainly due to interband transitions, which are not included in (18). As expected, the force is small for $E_{F}=0$ and is on the level of $F_{L}$ for the Fermi level $E_{F} \gtrsim \Delta$. Typically the force changes $3-5$ times on the interval $0<E_{F} \lesssim \Delta$ proving significant sensitivity to the Fermi level position.

The force for graphene-on-membrane is shown in fig. 2(b). The behavior is similar to that for suspended graphene. However, in this case the force has to be compared with the background force for membrane shown in fig. 2(c). The latter one was calculated using frequency-dependent dielectric functions of $\mathrm{SiO}_{2}$ and $\mathrm{Au}$. The relative force $\Delta F / F_{b}$ varies in the range $10-100 \%$; it is small for short separation and increases with $a$. The background force $F_{b}$ can be reduced further by decreasing thickness and/or the permittivity of the membrane.

Conclusions and discussion. - In this paper we analyzed the Casimir interaction of a graphene-covered dielectric with a metal plate. The dielectric function of graphene was found at finite temperature for imaginary frequencies for the material with a finite bandgap and non-zero Fermi level. A simple expression (3) describes the graphene contribution to the force. We can conclude that for graphene with the gap $2 \Delta \gg T$ there is a strong dependence of the Casimir force on both the temperature and the Fermi level. This is realized at distances $a \gg \hbar v_{F} / T$ when the main contribution to $\Delta F$ originates from thermal fluctuations. The predicted force is measurable with modern AFM instruments and can have significant technological applications. Graphene-on-membrane interacting with a metal has special interest for practical applications. This configuration combines mechanical strength with unique electronic properties of graphene. It allows tailoring of the Casimir force by electronic means. Manipulations with the thermal force opens up completely new possibilities which, so far, seemed to have pure academic interest for condensed matter. For example, it becomes possible to observe the non-equilibrium Casimir force $[35,36]$ between solid bodies at distances $\sim 100 \mathrm{~nm}$. This possibility put the Casimir effect on the same ground as the short distance radiative heat transfer [37]. For all bulk materials the equilibrium component of the force at $a \sim 100 \mathrm{~nm}$ is orders of magnitude larger than the non-equilibrium one. However, for suspended graphene or graphene-on-membrane interacting with a metal these components of the total force can be comparable.

\section{REFERENCES}

[1] Casimir H. B. G., Proc. K. Ned. Akad. Wet., 51 (1948) 793.

[2] Rodriguez A. W., Capasso F. and Johnson S. G., Nat. Photon., 5 (2011) 11.

[3] Dzyaloshinski I. E., Lifshitz E. M. and Pitaevski L. P., Adv. Phys., 38 (1961) 165.

[4] Iannuzzi D., Lisanti M. and Capasso F., Proc. Natl. Acad. Sci. U.S.A., 101 (2004) 4019.

[5] de Man S., Heeck K., Wijngaarden R. J. and IANnuzzi D., Phys. Rev. Lett., 103 (2009) 040402.

[6] Torricelli G., van Zwol P. J., ShPak O., Binns C., Palasantzas G., Kooi B. J., Svetovoy V. B. and Wuttig M., Phys. Rev. A, 82 (2010) 010101(R).

[7] Chen F., Klimchitskaya G. L., Mostepanenko V. M. and Mohideen U., Phys. Rev. B, 76 (2007) 035338.

[8] Obrecht J. M., Wild R. J., Antezza M., Pitaevski L. P., Stringari S. and Cornell E. A., Phys. Rev. Lett., 98 (2007) 063201.

[9] Sushkov A. O., Kim W. J., Dalvit D. A. R. and Lamoreaux S. K., Nat. Phys., 7 (2011) 230. 
[10] Novoselov K. S., Geim A. K., Morozov S. V., Jiang D., Zhang Y., Grigorieva I. V. and Firsov A. A., Science, 306 (2004) 666.

[11] Castro Neto A. H., Guinea F., Peres N. M. R., Novoselov K. S. and Geim A. K., Rev. Mod. Phys., 81 (2009) 109.

[12] Kotov V. N., Uchoa B., Pereira V. M., Castro Neto A. H. and Guinea F., arXiv:1012.3484.

[13] Bordag M., Geyer B., Klimchitskaya G. L. and Mostepanenko V. M., Phys. Rev. B, 74 (2006) 205431.

[14] Bordag M., Fialkovsky I. V., Gitman D. M. and Vassilevich D. V., Phys. Rev. B, 80 (2009) 245406.

[15] Sernelius Bo E., EPL, 95 (2011) 57000.

[16] Gómez-Santos G., Phys. Rev. B, 80 (2009) 245424.

[17] Boloting K. I., Sikes K. J., Jiang Z., Klima M., Fudenberg G., Hone J., Kim P. and Stormer H. L., Solid State Commun., 146 (2008) 351.

[18] Han M. Y., Ozyilimaz B., Zhang Y. and Kim P., Phys. Rev. Lett., 98 (2007) 206805.

[19] Zhou S. Y., Gweon G. H., Fedorov A. V., First P. N., De Heer W. A., Lee D. H., Guinea F., Castro Neto A. H. and Lanzara A., Nat. Mater., 6 (2007) 770.

[20] Yavari F., Kritzinger C., Gaire C., Song L., Gullapalli H., Borca-Tasciuc T., Ajayan P. M. and Koratkar N., Small, 6 (2010) 2535.

[21] Balog R., Jørgensen B., Nilsson L., Andersen M., Rienks E., Bianchi M., Fanetti M., LÆgsgaard E., Baraldi A., Lizzit S., Sluivancanin Z., Besenbacher F., Hammer B., Pedersen T. G., Hofmann P. and HornekÆr L., Nat. Mater., 9 (2010) 315.

[22] Park C.-H., Yang L., Son Y.-W., Cohen M. L. and Louie S. G., Nat. Phys., 4 (2008) 213.

[23] Bai J., Zhong X., Jiang S., Huang Y. and Duan X., Nat. Nanotechnol., 5 (2010) 190.
[24] Kim M., Safron N. S., Han E., Arnold M. S. and Gopalan P., Nano Lett., 10 (2010) 1125.

[25] Zhang L., Diao S., Nie Y., Yan K., Liu N., Dai B., Xie Q., Reina A., Kong J. and Liu Z., J. Am. Chem. Soc., 133 (2011) 2706.

[26] Falkovsky L. A. and Pershoguba S. S., Phys. Rev. B, 76 (2007) 153410.

[27] Stauber T., Peres N. M. R. and Geim A. K., Phys. Rev. B, 78 (2008) 085432.

[28] Nair R. R., Blake P., Grigorenko A. N., Noveselov K. S., Booth T. J., Stauber T., Peres N. M. R. and Geim A. K., Science, 320 (2008) 1308.

[29] Dawlaty J. M., Shivaraman S., Strait J., George P., Chandrashekhar M., Rana F., Spencer M. G., Veksler D. and Chen Y. Q., Appl. Phys. Lett., 93 (2008) 131905.

[30] Fialkovsky I. V., Marachevsky V. N. and Vassilevich D., Phys. Rev. B, 84 (2011) 035446.

[31] Stern F., Phys. Rev. Lett., 18 (1967) 546.

[32] Esquivel R. and Svetovoy V. B., Phys. Rev. A, 69 (2004) 062102.

[33] van der Zande A. M., Barto R. A., Alden J. S., Ruis-Vargas C. S., Whitney W. S., Pham P. H. Q., Park J., Parpia J. M., Craighead H. J. and McEuen P. L., Nano Lett., 10 (2010) 4869.

[34] Pyatkovskiy P. K., J. Phys.: Condens. Matter, 21 (2009) 025506.

[35] Antezza M., Pitaevskil L. P., Stringari S. and Svetovoy V. B., Phys. Rev. Lett., 97 (2006) 223203.

[36] Antezza M., Pitaevskit L. P., Stringari S. and Svetovoy V. B., Phys. Rev. A, 77 (2008) 022901.

[37] Rousseau E., Siria A., Jourdan G., Voltz S., Comin F., Chevrier J. and Greffet J. J., Nat. Photon., 3 (2009) 514. 